\title{
Pediatric Giant Cell Glioblastoma Presenting with Intracranial Dissemination at Diagnosis: A Case Report
}

\author{
Takamasa KinOSHITA, ${ }^{1}$ Hirohito YANO, ${ }^{1,2}$ Noriyuki NAKAYAMA, ${ }^{1}$ \\ Natsuko SuZUI, ${ }^{3}$ Tomohiro IIDA, ${ }^{1}$ Saori ENDO, ${ }^{4}$ Shiho YASUE, ${ }^{4}$ Michio OZEKI, ${ }^{4}$ \\ Kazuhiro KOBAYASHI, ${ }^{3}$ Tatsuhiko MIYAZAKI, ${ }^{3}$ and Toru IWAMA ${ }^{1}$ \\ ${ }^{1}$ Department of Neurosurgery, Gifu University Graduate School of Medicine, Gifu, Gifu, Japan \\ ${ }^{2}$ Chubu Medical Center for Prolonged Traumatic Brain Dysfunction, Kizawa Memorial \\ Hospital, Minokamo, Gifu, Japan \\ ${ }^{3}$ Department of Pathology, Gifu University Hospital, Gifu, Gifu, Japan \\ ${ }^{4}$ Department of Pediatrics, Gifu University Graduate School of Medicine, Gifu, Gifu, Japan
}

\begin{abstract}
Giant cell glioblastoma (GCG) is a rare subtype of glioblastoma multiforme (GBM), and it often occurs in younger patients; however, its onset in children is extremely noticeable. A 7-year-old girl presented with a headache and restlessness. A giant tumor that was $7 \mathbf{c m}$ in diameter was found by magnetic resonance imaging (MRI) in the left frontal lobe with intracranial dissemination. Because the tumor had extended to the lateral ventricles and occluded the foramen of Monro causing hydrocephalus, she underwent ventricular drainage and neuro-endoscopic biopsy from the left posterior horn of the lateral ventricle. The initial pathological diagnosis was an atypical teratoid/rhabdoid tumor (AT/RT). When the dissemination subsided after the first chemotherapy with vincristine, doxorubicin, and cyclophosphamide, she underwent the first tumor resection via a left frontal transcortical approach. After surgery, the second chemotherapy with ifosfamide, cisplatin, and etoposide was not effective for the residual tumor and intracranial dissemination. The second surgery via a transcallosal approach achieved nearly total resection leading to an improvement of the hydrocephalus. The definitive pathological diagnosis was GCG. Despite chemo-radiation therapy, the dissemination in the basal cistern reappeared and the hydrocephalus worsened. She was obliged to receive a ventriculo-peritoneal (VP) shunt and palliative care at home; however, her poor condition prevented her discharge. Ten months after admission, she died of tumor progression. The peritoneal dissemination was demonstrated by cytology of ascites. In conclusion, although unusual, pediatric GCG may be disseminated at diagnosis, in which case both tumor and hydrocephalus control need to be considered.
\end{abstract}

Keywords: giant cell glioblastoma, pediatrics, dissemination, ventriculo-peritoneal shunt, immunohistochemistry

\section{Introduction}

Malignant gliomas such as glioblastoma multiforme (GBM) are relatively rare in children compared to adults. ${ }^{1)}$ Pediatric GBMs constitute approximately $3 \%$ of all central nervous system tumors in children $^{2)}$; however, only $0.6 \%-7.9 \%$ of all GBMs occur

Received April 29, 2020; Accepted July 8, 2020

Copyright $\subseteq 2021$ by The Japan Neurosurgical Society This work is licensed under a Creative Commons AttributionNonCommercial-NoDerivatives International License. during childhood. ${ }^{3)}$ More than $70 \%$ of pediatric GBMs occur in the second decade; thus, pediatric GBMs in the first decade are especially unusual. ${ }^{4)}$

Giant cell glioblastoma (GCG) is an unusual GBM subtype defined by the latest classification of the World Health Organization. ${ }^{5,6)}$ GCG constitutes only $1 \%$ of adult GBMs and $3 \%$ of pediatric GBMs. ${ }^{6,7)}$ Although GCGs are diagnosed at relatively younger ages than the more common $\mathrm{GBM},{ }^{7}$ they rarely develop in childhood.

We present a case of pediatric GCG with intracranial dissemination, which is extremely rare with respect to age and presence of dissemination at 
diagnosis. We discuss the radiological diagnosis, treatment, and pathological examination of this disease.

\section{Case Report}

\section{History and preoperative examination}

A 7-year-old Japanese girl presented with complaints of headaches since the age of 3 years. One month prior to admission, she had posterior neck pain and loss of appetite; subsequently, she visited a nearby hospital. A cervical magnetic resonance imaging (MRI) examination did not reveal any significant changes (Fig. 1A). However, 1 week later, she complained of a headache and loss of appetite again. She then had a head MRI examination, which showed a left frontal mass that was $7 \mathrm{~cm}$ in diameter with dissemination into the left lateral ventricular wall and the basal cistern (Figs. 1B-1D).

She did not have any previous developmental disorders or other medical history. When she was admitted to our hospital, the patient's Glasgow Coma Scale score was 10 (Eyes $[E]=2$, Verbal $[V]=4$, Motor $[\mathrm{M}]=4$ ), but she had no apparent motor weakness. Gadolinium-enhanced head MRI revealed the heterogeneously enhanced tumor in the left frontal lobe extending to the lateral ventricles with acute hydrocephalus due to obstruction of the foramen of Monro. At first, the patient underwent ventricular drainage and a neuro-endoscopic biopsy via the left posterior horn of the lateral ventricle. The initial pathological diagnosis was an atypical teratoid/rhabdoid tumor (AT/RT). Fortunately, the first 14-day chemotherapy cycle with vincristine $\left(1.5 \mathrm{mg} / \mathrm{m}^{2}\right)$, doxorubicin $(37.5$ $\mathrm{mg} / \mathrm{m}^{2}$ ), and cyclophosphamide $\left(1800 \mathrm{mg} / \mathrm{m}^{2}\right)$ (VDC) reduced the intracranial dissemination. Next, she underwent the first tumor resection via a left frontal transcortical approach. Afterward, she underwent a second 21-day chemotherapy cycle with ifosfamide $\left(2000 \mathrm{mg} / \mathrm{m}^{2}\right)$, carboplatin $\left(500 \mathrm{mg} / \mathrm{m}^{2}\right)$, and etoposide $\left(100 \mathrm{mg} / \mathrm{m}^{2}\right)$ (ICE); however, the residual tumor showed no changes. Instead, the hydrocephalus worsened, and the dissemination was newly detected in the spinal region (Fig. 2) and cerebellopontine angle. Approximately 1.5 months after the first resection, she underwent a second tumor resection via a transcallosal approach, and a nearly total resection was achieved, resulting in improvement of the hydrocephalus. Then, the patient received radiation therapy: 23.4 Gy for the craniospinal lesion, 30 Gy for the tumor cavity, and 29.6 Gy for the cerebellopontine lesion. Four cycles of weekly vincristine therapies $\left(1.5 \mathrm{mg} / \mathrm{m}^{2}\right)$ and a second VDC therapy were not effective. After the patient was diagnosed with GCG by the Central Pathological Diagnosis of the Department of Pathology, National

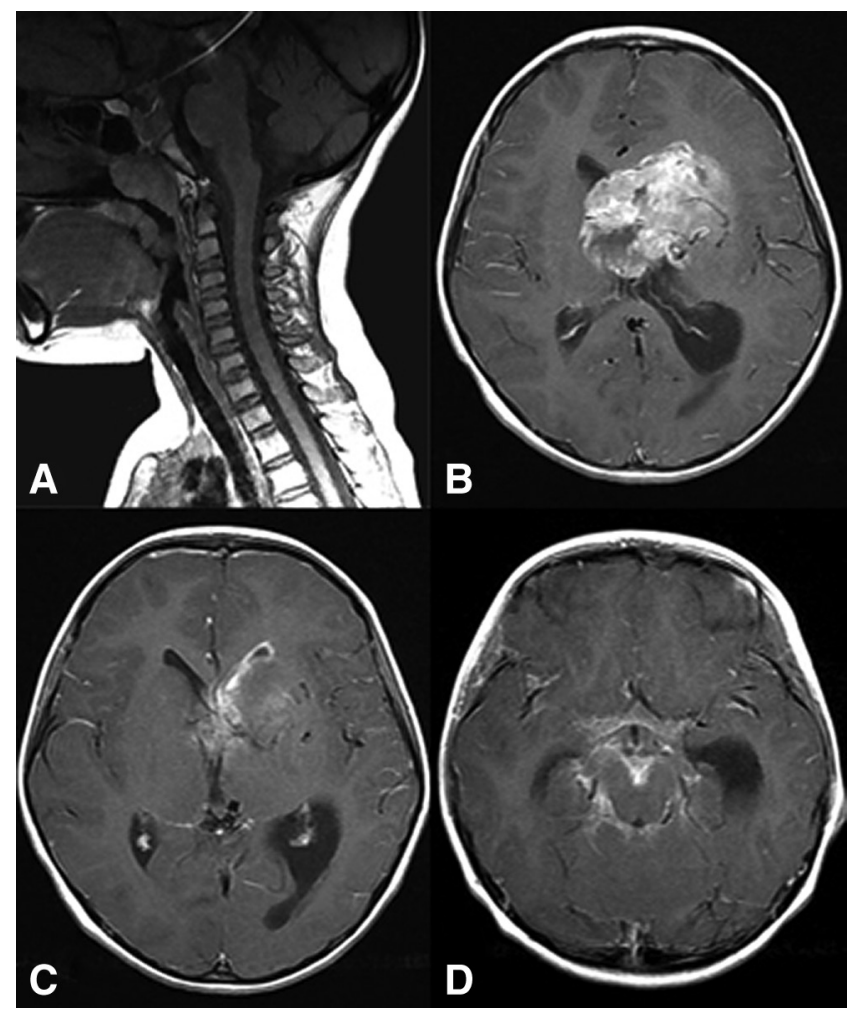

Fig. 1 (A) Plain cervical MRI scan performed at the previous hospital showing no abnormal findings. (B-D) Initial contrast-enhanced head MRI scan showing a homogeneously enhanced 7 -cm left frontal mass lesion (A), and dissemination into the left lateral ventricular wall (B) and the basal cistern (C). MRI: magnetic resonance imaging.

Center for Child Health and Development, she received chemotherapy with five cycles of temozolomide $\left(150 \mathrm{mg} / \mathrm{m}^{2}\right)$ and four cycles of bevacizumab (10 mg/kg), which unfortunately did not help to control the dissemination. As the hydrocephalus worsened, she was obliged to undergo a ventriculoperitoneal (VP) shunt instead of ventricular drainage for subsequent postoperative palliative home care. Eventually, she could not be discharged home because of anuresis and oral feeding difficulties. Two months after the VP shunt, she experienced severe abdominal distension, followed by dyspnea. Ten months after admission, she died of respiratory failure caused by tumor progression. The peritoneal dissemination was demonstrated by cytology of ascites.

\section{Histological examination}

Hematoxylin-eosin staining of the first surgical specimens showed giant cells consisting mainly of a mega nucleus and a bizarre polynucleus, which was sometimes located eccentrically, as well as cells with a wide range of eosinophilic, vacuolated, and 


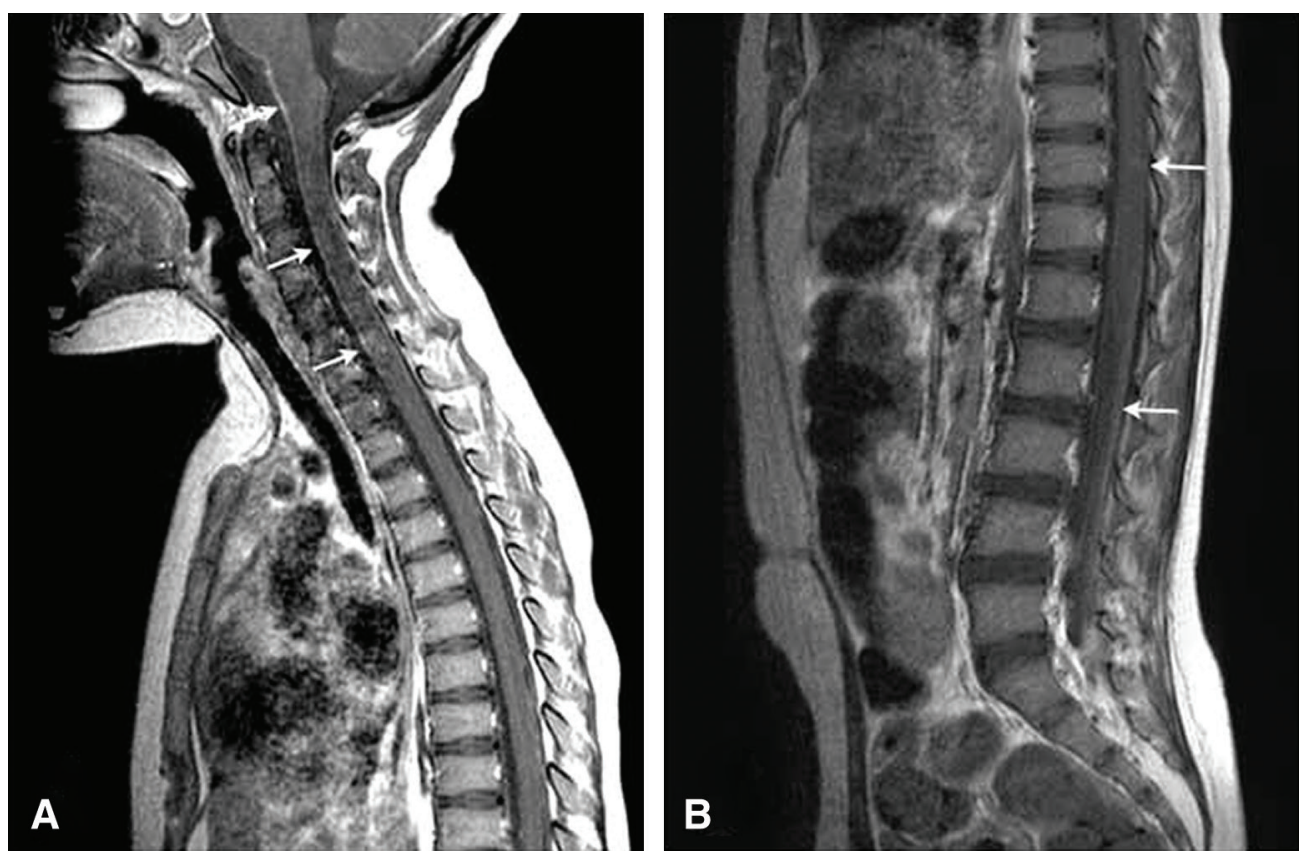

Fig. 2 Gadolinium-enhanced spinal MRI scan showing new disseminations (arrow) after the first tumor resection in the cervical (A) and lumbar (B) regions. MRI: magnetic resonance imaging.

foamy cytoplasm (Fig. 3A). Tumors consisted of small to medium spindle-shaped or multi-ridged cells, cells with multipolar processes, and small cells with a high nuclear-to-cytoplasm ratio (Fig. 3B). These cells were diffusely and densely proliferating. Many smallto medium-sized cells undergoing mitosis were observed, but only a few giant cells undergoing mitosis were observed. Hypervascularization of small vessels with congestion was observed, but no microvascular proliferation was detected. In some areas, geographic necroses were observed.

Immunohistochemistry showed diffuse positive nuclear staining for p53 (1:200, Dako, Fig. 3C) and INI-1 (1:500, Becton Dickinson, Fig. 3D), and cytoplasmatic staining for vimentin (1:200, Leica, Fig. 3E). No immunostaining was detected for IDH1R132H (1:100, Dianova, Fig. 3F), H3K27M (1:200, Abcam, Fig. 3G), and glial fibrillary acidic protein (GFAP) (1:100, Dako, Fig. 3H). MIB-1 immunostaining (1:200; Dako, Fig. 3I) was positive in $30 \%$ of giant cells and $60 \%$ of small cells. In addition, using the Pyrosequencing method from the Japan Children's Cancer Group, we obtained the results that both H3F3A K27M and H3F3A G34 were wildtype. When taken together, this case was IDH-wildtype, p53 mutant, and H3F3A K27M and H3F3A G34 wildtype. These findings were considered to correspond to a diagnosis of GCG. Immunohistochemistry for PTEN and CD133 was performed to investigate factors related to intracranial dissemination in the early stage. As a result, PTEN (1:10000; proteintech, Fig. 3J) was strongly positive in the cytoplasm of the tumor cells; therefore, PTEN mutation was denied. In contrast, moderate CD133 expression (1:1500; proteintech, Fig. 3K) was observed in not only giant cells but also small cells. Papanicolaou staining for the ascites collected by peritoneal puncture revealed a lot of small oval atypical cells rather than giant cells (Fig. 3L).

\section{Discussion}

GCG is included in the World Health Organization classification as a rare subtype of GBM. ${ }^{6)}$ The mean age of onset for GCG is lower than that for GBM, and GCG commonly localizes to the frontal and temporal lobes. ${ }^{7-9)}$ The average age at diagnosis is 59 years for GBM and 44 years for GCG. ${ }^{10)}$ Therefore, the present case represents a very young case in the GCG group. The H3K27M mutation is frequent in diffuse intrinsic pontine glioma and non-brain stem midline tumor, while G34R/V mutation occurs in pediatric high-grade glioma (HGG) of the cerebral cortex. ${ }^{11)}$ It was reported that the former occurred mainly in younger patients (median age 11 years), whereas the latter occurred in older patients (median age 20 years). ${ }^{12)}$ Accordingly, in terms of age and site of origin, there is no contradiction that both mutations were wildtype in this case. Based on molecular diagnosis, p53 

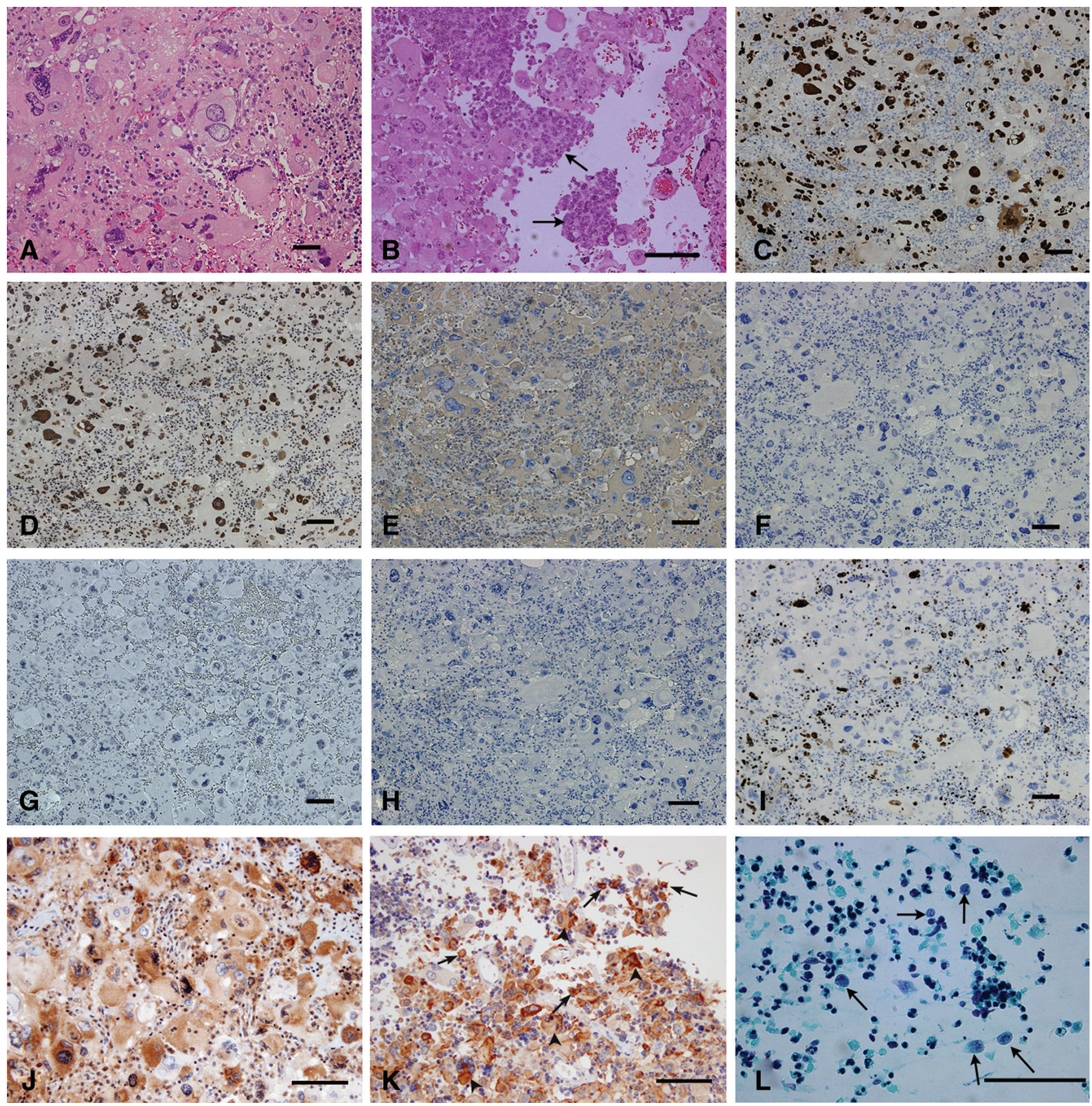

Fig. 3 (A) Hematoxylin-eosin staining of the first surgical specimens showing giant cells mainly consisting of a mega nucleus and a bizarre polynucleus, which was sometimes located eccentrically, and cells with a wide range of eosinophilic, vacuolated, and foamy cytoplasm. (B) Hematoxylin-eosin staining showing clusters of small oval cells. (C-K) Immunohistochemical staining for the first surgical specimens. (C) Diffuse positivity in the nuclei for p53. (D) Diffuse positivity in the nuclei for INI1. (E) Diffuse positivity in the cytoplasm for vimentin. (F) Negative staining for IDH1R132H. (G) Negative staining for H3K27M. (H) Negative staining for GFAP. (I) Positive staining for MIB-1 in 30\% of giant cells. (J) Positive staining for PTEN in the cytoplasm of giant cells. (K) Positive staining for CD133 in both giant (arrowheads) and small cells (arrows), contrast negative findings in endothelial cells and inflammatory cells. (L) Papanicolaou staining for the ascites collected by peritoneal puncture at the time of death showing some atypical oval cells with intense hematoxylin staining (arrows); these cells resemble small cells shown in B and K. Scale bar indicates $100 \mu \mathrm{m}$. GFAP: glial fibrillary acidic protein. 
mutation $^{13-16)}$ and PTEN mutation ${ }^{13,17)}$ were reported to be $75-90 \%$ and $14-31.3 \%$ in GCG, respectively. Therefore, it seems that this case is consistent with the diagnosis.

According to several reports, the prognosis of GCG is significantly better than that of GBM..$^{1,7,18-20)}$ The median overall survival has been reported to be 14.2-15.5 months for GCG and 11.7-13.0 months for GBM. ${ }^{7,20)}$ Adult and pediatric patients with GCG have been reported to survive longer compared to those with GBM; ${ }^{21-28)}$ however, this finding was not supported by a pediatric series reported by Karremann et al. ${ }^{20)}$ Additionally, gross total resection has been reported as a positive prognostic clinical parameter in pediatric patients with GCG. ${ }^{20)}$ Several studies have shown that adjuvant radiotherapy and chemotherapy result in improvements of prognosis. ${ }^{7,29)}$ However, the overall survival and prognostic factors of GCG with dissemination at diagnosis are not well known. Vertosick et al. ${ }^{30)}$ reported that neuroaxis dissemination was found in 11 of 600 patients $(1.8 \%)$ treated for GBM at the University of Pittsburg. Furthermore, Benesch et al. reported 10 of 324 patients $(3.1 \%)$ with HGG having primary tumor dissemination. ${ }^{31)}$ Arita et al. ${ }^{32)}$ identified $14 \%$ of patients with malignant glioma with leptomeningeal dissemination, in which only one patient had dissemination at the time of the initial presentation. Thus, GCG with intracranial dissemination at diagnosis is very rare. Onda et al. ${ }^{33)}$ have suggested that less-differentiated tumor cells and a lower rate of GFAP expression contribute to a higher tendency of cells to spread into the cerebrospinal fluid, which is in accordance with the GFAP negativity and intracranial dissemination at an early stage observed in the present case. In H3K27M mutant GBM, 28\% of cases were reported to contain giant cells, whereas most wildtype GBM contain monomorphic small, round cells. ${ }^{34)}$ These pathological findings are consistent with those in this case of H3K27M wildtype GBM. It was reported that p53 mutation was found in $63.3 \%$ of $\mathrm{H} 3 \mathrm{~K} 27 \mathrm{M}$ wildtype GBM and that the p53 mutant cell lines represent a more aggressive tumor that promotes proliferation, migration, and invasion of GBM cells in vitro. ${ }^{35,36)}$ Furthermore, high CD133 expression is reported to be a useful predictor for GBM dissemination. ${ }^{5,37)}$ It was also reported that PTEN might be one of the suppressor factors in glioma cell dissemination, since PTEN mutation was significantly detected in malignant gliomas with meningeal gliomatosis. ${ }^{38,39)}$ However, PTEN mutation was not demonstrated in our case. High MIB-1 labeling index (LI; $\geq 35 \%$ ) was also reported to be associated with GBM dissemination. ${ }^{39}$ The MIB-1 LI of up to $60 \%$ in our case might have been a factor associated with dissemination. Altogether, the factors considered to be related to intracranial dissemination in this case were GFAP-negative, p53 mutation, CD 133-positive, and high MIB1-LI.

Korshunov et al. ${ }^{40)}$ reported that $>90 \%$ of cases displaying tumor dissemination through the central nervous system at recurrence were found in the high-risk group, which supports the very poor prognosis of the present case of GCG with initially presenting dissemination. In contrast, Benesch et al. ${ }^{31}$ ) reported that the median overall survival was not significantly different between pediatric patients with and without dissemination of HGG. However, because the prognosis cannot be improved only by removing the tumor mass lesion, a treatment strategy for dissemination is needed from an early stage of therapy. Accordingly, we performed a first round of chemotherapy (VDC therapy) before the first craniotomy, followed by a second round (ICE therapy) and a second craniotomy. The chemotherapies with VDC and ICE were chosen based on the initial diagnosis of AT/RT. The former showed a partial response for dissemination while the latter was ineffective. Gross total resection was achieved by the two-staged surgery, and the final pathological diagnosis was changed to GCG. When we changed the chemotherapy treatment to temozolomide, we had difficulty in controlling the dissemination. As a result, the hydrocephalus worsened, and the patient could not continue with the ventricular drainage. Then, we performed a VP shunt so that the patient could receive palliative care at home. We repeatedly examined the cytology of the cerebrospinal fluid collected from the ventricular drainage before the VP shunt surgery, because of a previously reported brain tumor seeding following shunt placement. ${ }^{41)}$ Unfortunately, a small number of atypical cells were detected in the cytology immediately before the shunt placement, but surgery was performed because of the patient's limited life expectancy. As a result, the headache subsided; however, the patient's level of consciousness worsened, and abdominal distension occurred 1 month after the shunt placement. The cytology of ascites did not reveal any giant cells, but some small oval atypical cells, which resembled the cells observed by hematoxylin-eosin staining in the first surgical specimens. Hoffmann et al. ${ }^{42}$ used a Millipore filter encased in Rickham reservoir for VP shunt to avoid pleural metastasis. The use of such a filter is likely to cause obstruction of the shunt tube, but may be necessary to prevent pleural metastasis, since a VP shunt may be a potential route for iatrogenic metastasis. ${ }^{43)}$ 


\section{Conclusion}

We describe an extremely rare case of GCG as it developed at a very early age, where the patient presented with intracranial dissemination at the time of diagnosis. Even though GCG has a better outcome than GBM, the prognosis for GCG with dissemination is generally poor. When VP shunt placement is performed to treat hydrocephalus, the possibility of pleural metastasis should be monitored closely, and the timing of the surgery could play a role in palliative care.

\section{Acknowledgments}

We would like to thank Dr. Ran Tomomasa, Dr. Sumihito Nobusawa, and Dr. Junko Hirato of the Department of Pathology, National Center for Child Health and Development, for their pathological diagnosis and comments.

We thank the Japan Children's Cancer Group for their support in diagnosis.

We thank all staff of the Department of Pathology at Gifu University Hospital for their technical support with the pathological diagnosis.

\section{Conflicts of Interest Disclosure}

Dr. T. Iwama received a research grant from Ogaki Tokushukai Hospital and GlaxoSmithKline K.K. in 2020. The grant was not used for funding this study. The remaining authors declare no conflicts of interest.

\section{References}

1) Biswas R, Gupta S, Haresh KP, Halder A, Rath GK: Giant cell glioblastoma with spinal and spinal leptomeningeal metastasis in a child: A rare presentation of a rare tumor. J Craniovertebr Junction Spine 9: 202-204, 2018

2) Song KS, Phi JH, Cho BK, et al.: Long-term outcomes in children with glioblastoma. J Neurosurg Pediatr 6: 145-149, 2010

3) Zipp L, Schwartz KM, Hewer E, Yu Y, Stippich C, Slopis JM: Magnetic resonance imaging and computed tomography findings in pediatric giant cell glioblastoma. Clin Neuroradiol 22: 359-363, 2012

4) Das KK, Mehrotra A, Nair AP, et al.: Pediatric glioblastoma: clinico-radiological profile and factors affecting the outcome. Childs Nerv Syst 28: 2055-2062, 2012

5) Ogawa K, Kurose A, Kamataki A, Asano K, Katayama $\mathrm{K}$, Kurotaki H: Giant cell glioblastoma is a distinctive subtype of glioma characterized by vulnerability to DNA damage. Brain Tumor Pathol 37: 5-13, 2020

6) Ohgaki H, Kleuhues P, Plate KH, Nakazato Y, Binger DD: Giant cell glioblastoma, in Louis DN, Ohgaki H, Wiestler OD, et al. (eds): World Health Organization
Classification of Tumours of the Central Nervous System, Lyon, IARC, 2016, pp 46-47

7) Jin $\mathrm{MC}, \mathrm{Wu} \mathrm{A}$, Xiang $\mathrm{M}$, et al.: Prognostic factors and treatment patterns in the management of giant cell glioblastoma. World Neurosurg 128: e217-e224, 2019

8) Kozak KR, Moody JS: Giant cell glioblastoma: a glioblastoma subtype with distinct epidemiology and superior prognosis. Neuro Oncol 11: 833-841, 2009

9) De Prada I, Cordobés F, Azorín D, Contra T, Colmenero I, Glez-Mediero I: Pediatric giant cell glioblastoma: a case report and review of the literature. Childs Nerv Syst 22: 285-289, 2006

10) Oh JE, Ohta T, Nonoguchi N, et al.: Genetic alterations in gliosarcoma and giant cell glioblastoma. Brain Pathol 26: 517-522, 2016

11) Gianno F, Antonelli M, Ferretti E, Massimino M, Arcella A, Giangaspero F: Pediatric high-grade glioma: a heterogeneous group of neoplasms with different molecular drivers. Glioma 1: 117-124, 2018

12) Schwartzentruber J, Korshunov A, Liu XY, et al.: Driver mutations in histone H3.3 and chromatin remodelling genes in paediatric glioblastoma. Nature 482: 226-231, 2012

13) Peraud A, Watanabe K, Schwechheimer K, Yonekawa Y, Kleihues P, Ohgaki H: Genetic profile of the giant cell glioblastoma. Lab Invest 79: 123-129, 1999

14) Martinez R, Roggendorf W, Baretton G, et al.: Cytogenetic and molecular genetic analyses of giant cell glioblastoma multiforme reveal distinct profiles in giant cell and non-giant cell subpopulations. Cancer Genet Cytogenet 175: 26-34, 2007

15) Meyer-Puttlitz B, Hayashi Y, Waha A, et al.: Molecular genetic analysis of giant cell glioblastomas. Am J Pathol 151: 853-857, 1997

16) Peraud A, Watanabe K, Plate KH, Yonekawa Y, Kleihues P, Ohgaki H: p53 mutations versus EGF receptor expression in giant cell glioblastomas. J Neuropathol Exp Neurol 56: 1236-1241, 1997

17) Duerr EM, Rollbrocker B, Hayashi Y, et al.: PTEN mutations in gliomas and glioneuronal tumors. Oncogene 16: 2259-2264, 1998

18) Belsuzarri TA, Araujo JF, Catanoce AP, et al.: Giant cells glioblastoma: case report and pathological analysis from this uncommon subtype of glioma. Rare Tumors 7: 5634, 2015

19) Bin Abdulrahman AK, Bin Abdulrahman KA, Bukhari YR, Faqihi AM, Ruiz JG: Association between giant cell glioblastoma and glioblastoma multiforme in the United States: a retrospective cohort study. Brain Behav 9: e01402, 2019

20) Karremann M, Butenhoff S, Rausche U, Pietsch T, Wolff JE, Kramm CM: Pediatric giant cell glioblastoma: new insights into a rare tumor entity. Neuro Oncol 11: 323-329, 2009

21) Akslen LA, Mørk SJ, Larsen JL, Myrseth E: Giant cell glioblastoma: a work-up of 2 cases with long survival. Acta Neurol Scand 79: 194-199, 1989

22) Margetts JC, Kalyan-Raman UP: Giant-celled glioblastoma of brain. A clinico-pathological and radiological 
study of ten cases (including immunohistochemistry and ultrastructure). Cancer 63: 524-531, 1989

23) Sabel M, Reifenberger J, Weber RG, Reifenberger G, Schmitt HP: Long-term survival of a patient with giant cell glioblastoma. Case report. J Neurosurg 94: 605-611, 2001

24) Raco A, Bristot R, Salvati M, Delfini R: Malignant supratentorial astrocytomas of late childhood. Our experience with 25 cases. Childs Nerv Syst 13: 341-344, 1997

25) Kroh H, Matyja E, Marchel A, Bojarski P: Heavily lipidized, calcified giant cell glioblastoma in an 8-yearold patient, associated with neurofibromatosis type 1 (NF1): report of a case with long-term survival. Clin Neuropathol 23: 286-291, 2004

26) Klein R, Mölenkamp G, Sörensen N, Roggendorf W: Favorable outcome of giant cell glioblastoma in a child. Report of an 11-year survival period. Childs Nerv Syst 14: 288-291, 1998

27) Artico M, Cervoni L, Celli P, Salvati M, Palma L: Supratentorial glioblastoma in children: a series of 27 surgically treated cases. Childs Nerv Syst 9: 7-9, 1993

28) Deb P, Sharma MC, Chander B, Mahapatra AK, Sarkar C: Giant cell glioblastoma multiforme: report of a case with prolonged survival and transformation to gliosarcoma. Childs Nerv Syst 22: 314-319, 2006

29) Oh T, Rutkowski MJ, Safaee M, et al.: Survival outcomes of giant cell glioblastoma: institutional experience in the management of 20 patients. J Clin Neurosci 21: 2129-2134, 2014

30) Vertosick FT, Selker RG: Brain stem and spinal metastases of supratentorial glioblastoma multiforme: a clinical series. Neurosurgery 27: 516-521; discussion 521-522, 1990

31) Benesch M, Wagner S, Berthold F, Wolff JE: Primary dissemination of high-grade gliomas in children: experiences from four studies of the Pediatric Oncology and Hematology Society of the German Language Group (GPOH). J Neurooncol 72: 179-183, 2005

32) Arita N, Taneda M, Hayakawa T: Leptomeningeal dissemination of malignant gliomas. Incidence, diagnosis and outcome. Acta Neurochir (Wien) 126: 84-92, 1994

33) Onda K, Tanaka R, Takahashi H, Takeda N, Ikuta F: Cerebral glioblastoma with cerebrospinal fluid dissemination: a clinicopathological study of 14 cases examined by complete autopsy. Neurosurgery 25: 533-540, 1989
34) Neumann JE, Dorostkar MM, Korshunov A, et al.: Distinct histomorphology in molecular subgroups of glioblastomas in young patients. J Neuropathol Exp Neurol 75: 408-414, 2016

35) Bozkurt SU, Dagcinar A, Tanrikulu B, et al.: Significance of H3K27M mutation with specific histomorphological features and associated molecular alterations in pediatric high-grade glial tumors. Childs Nerv Syst 34: 107-116, 2018

36) Olafson LR, Gunawardena M, Nixdorf S, McDonald KL, Rapkins RW: The role of TP53 gain-of-function mutation in multifocal glioblastoma. J Neurooncol 147: 37-47, 2020

37) Sato A, Sakurada K, Kumabe T, et al.: Association of stem cell marker CD133 expression with dissemination of glioblastomas. Neurosurg Rev 33: 175-183; discussion 183-184, 2010

38) Izumoto S, Ohnishi T, Kanemura H, et al.: PTEN mutations in malignant gliomas and their relation with meningeal gliomatosis. J Neurooncol 53: 21-26, 2001

39) Kato H, Fujimura M, Kumabe T, Ishioka C, Kanamaru R, Yoshimoto T: PTEN gene mutation and high MIB1 labeling index may contribute to dissemination in patients with glioblastoma. J Clin Neurosci 11: 37-41, 2004

40) Korshunov A, Ryzhova M, Hovestadt V, et al.: Integrated analysis of pediatric glioblastoma reveals a subset of biologically favorable tumors with associated molecular prognostic markers. Acta Neuropathol 129: 669-678, 2015

41) Xu K, Khine KT, Ooi YC, Quinsey CS: A systematic review of shunt-related extraneural metastases of primary central nervous system tumors. Clin Neurol Neurosurg 174: 239-243, 2018

42) Hoffman HJ, Hendrick EB, Humphreys RP: Metastasis via ventriculoperitoneal shunt in patients with medulloblastoma. J Neurosurg 44: 562-566, 1976

43) Ingold B, Moschopulos M, Hutter G, et al.: Abdominal seeding of an atypical teratoid/rhabdoid tumor of the pineal gland along a ventriculoperitoneal shunt catheter. Acta Neuropathol 111: 56-59, 2006

Corresponding author: Hirohito Yano, MD, PhD Chubu Medical Center for Prolonged Traumatic Brain Dysfunction, Kizawa Memorial Hospital, 630 ShimoKobi, Kobi-Cho, Minokamo, Gifu 505-0034, Japan. e-mail: hyano-nsu@umin.ac.jp 